\title{
Thermal sintering of printable copper for enhanced conductivity of FTO coated glass substrates
}

Authors : Bahaa Abbas, Youmna Mohammad, Eifion Jewell* \& Justin Searle

Affiliation : SPECIFC, College of Engineering, Swansea University.

*Contact author : e.jewell@swansea.ac.uk

Keywords : nano copper; conductive ink; thermal sintering; FTO coated glass; transparent electrode manufacture.

\begin{abstract}
Copper inks potentially provide a cost effective replacement to silver for printed electronic circuits. In glass based applications such as PV or smart glass, it can provide a means of conductivity enhancement or additional functionality. Three inks consisting of a mixture of nano and micro copper particles were systematically studied to examine the relationship between sintering temperature, sintering time and gaseous environment on the electrical qualities of the sintered printed films deposited on FTO coated glass. There is a definite interaction between the particulate nature of the ink, the sintering conditions and the conductive properties of the film. Films containing only nano-particles provide the most conductive films with optimum sintering conditions of temperatures of $225^{\circ} \mathrm{C}$ for 60 minutes. The inclusion of micro particles increased the ideal sintering temperature but lowered the sintering time. An ink containing an equal mixture of nano and micro particles exhibited the lowest performance and this could be attributed to partial oxidation of the nano-particles along the conductive path, which occurs as a result of the presence of the micro particles.
\end{abstract}

\section{Introduction}

In the last decades, the synthesis of using copper nanoparticles ink has attracted research attention in the area of manufacturing conductive circuits, because of the promising potential of replacing silver nanoparticles ink which potentially leads to lower cost products. The conductive circuit is the basic fundamental part of any electrical / electronic device and while silver has excellent conductivity, is stable in organic suspensions and has good lifetime it is not only expensive but also subject to fluctuations in price. Numerous applications for copper ink have already been proven, these include RFID aerials [1], hybrid silicon / printed flexible circuits [2] and thin film transistor electrodes [3]. Each of these applications focussed on polymer substrates, most notably polyimide because of its ability to withstand higher sintering temperatures, product flexibility and its subsequent compatibility with reel to reel processes.

Copper inks can be classified into four main groups; thermal decomposition of soluble copper salts [4], [5], Copper oxide / hydroxide nano particles which are chemically / thermally reduced to copper [6], copper nano / micro particles which can sintered [7] and hybrid inks which use a combination of these technologies [8], [9]. The main challenge with using copper nanoparticles is the oxidation 
during sintering, as nano-copper is readily oxidized under temperatures over $130^{\circ} \mathrm{C},[10],[11]$. Proprietary copper inks overcome this problem with an organic coating (capping agent) which protects the reactive copper during suspension, deposition and drying but which undergoes decomposition / sublimation during the sintering process creating a conducting copper network of linked particles [12], [13]. The particles are linked by copper-copper interfaces which are less susceptible to oxidation as a result of their lower surface area / volume ratio. Although nano particles are preferred for their lower sintering temperatures, they have an appreciable cost penalty associated with manufacturing in controlled argon atmospheric conditions and filtering. There is therefore an economic driver for the move to mixed micro / nano copper systems.

Photonic sintering has proven popular due to its rapid processing speed. Laser sintering has been proven [14]-[16] as has broad spectrum intense white light photonic sintering [9], [17]-[19]. Multi spectrum photonic sintering (IR and white light) [20], [21] also allows for rapid sintering under ambient atmospheric conditions on flexible substrates. All these have been shown to be highly sensitive to the circuit pattern, film thickness, ink composition, substrate and other physical and optical properties. Substrates with high thermal mass act as a thermal sink, retarding the rapid sintering process, are particularly problematic and are less suited to photonic sintering. Thus, thermal sintering provides a more repeatable and consistent process but where the public domain science and technology is more scant.

Haque et al [22] demonstrated that the effectiveness to thermal sintering of nano copper ink was dictated by time, temperature and reducing atmosphere and could achieve between 3 and 4 times bulk resistivity when sintered under pure hydrogen environment at $250{ }^{\circ} \mathrm{C}$; such conditions are not industrially attractive from a safety perspective. Joo et al obtained a resistivity which was at best around $8 \times$ bulk [23] under pure nitrogen conditions for 1 hour. Kim et al achieved $12 x$ bulk resistivity in 40 minutes under $3 \% \mathrm{H}_{2}$ at $300{ }^{\circ} \mathrm{C}$, with the inclusion of a binder significantly reducing the conductivity achieved, [1].

FTO (Fluorine doped tin oxide) coated glass offers a low cost alternative transparent conductive substrate to ITO (indium tin oxide) coated glass and has been widely adopted by the third generation PV community for organic and perovskite (particularly carbon rear electrode) technologies [24], [25]. These PV technologies have transitioned to the module scale where larger dimensions demand enhanced conductivity of transparent electrodes by printing fine electrode structures between higher transparency and low conductivity FTO for more efficient charge extraction, [26]. This concept currently proposes silver but the deposition of printed copper inks for the bus bars and fine charge extraction electrodes within the structure would further reduce the bill of materials through silver ink elimination and thinner and more transparent FTO [27], [28].

The manufacturing process for perovskite PV requires several high temperature sintering / burn off stages and thus a thermal processing route for copper sintering is advantageous in terms of capital equipment. It was with this application in mind, that the investigation was initiated.

The overall aim of the study was to evaluate the performance of the copper inks printed to FTO conductive glass substrates. Specifically it aimed to identify and understand the relationship and interactions between the final electrical performance of the printed film and the copper ink characteristics, sintering time, sintering temperature and the atmosphere required for sintering. The 
findings would help identify processing productivity and capital / safety requirements in the transparent electrode manufacture.

\section{Method}

Three copper materials were sourced from Intrinsiq materials, these were labelled 100N, 50N50M \& 20 N80M in accordance with the proportion of nano $(\mathrm{N})$ and micro $(\mathrm{M})$ particle . Each represents the state of the art in nano copper screen printing materials. Each has been formulated such that they represent a range of pure nano copper and micro-nano particles. Rheology was measured using a Brookfiled RS/ 2 rheolometer operating in a controlled stress mode, while the material composition ratio was measured using Perkin-Elmer Pyrus 1 - TGA operating in a nitrogen atmosphere. Particle size distributions were measured on the dry but cured film using a 7800 JEOL SEM equipped with an InLens detector operating at a $10 \mathrm{kV}$ accelerating voltage. Images were subsequently analysed using ImageJ to ascertain particle sizes. All three inks exhibit similar thermal behaviour where there is a gradual reduction in mass in the $30-80^{\circ} \mathrm{C}$ temperature range due to solvent evaporation, with a rapid reduction in the mass as the rate of solvent loss increases as it nears they boiling point (Table 1), Figure $1(\mathrm{a}, \mathrm{b})$. For the $100 \mathrm{~N}$ ink, the rate of mass loss reduces significantly as the solvent is lost with little change in the $150-275^{\circ} \mathrm{C}$ range. Beyond $275^{\circ} \mathrm{C}$, there is a further mass loss which has been associated with binder burn off. Between $170^{\circ} \mathrm{C}$ and $275^{\circ} \mathrm{C}$, the two micro particle inks exhibit binder loss, with peaks in binder loss of $228^{\circ} \mathrm{C}$ and $192^{\circ} \mathrm{C}$ for the $50 \mathrm{~N} 50 \mathrm{M}$ and $20 \mathrm{~N} 80 \mathrm{M}$ inks respectively. The final material properties are summarized in Table 1 and Figure 1 with the copper content being calculated by determined by the final mass after a 1 hour residence time at $350{ }^{\circ} \mathrm{C}$.

Table 1 : Copper ink characteristics

\begin{tabular}{|l|c|c|c|}
\hline & $100 \mathrm{~N}$ & $50 N 50 \mathrm{M}$ & $20 \mathrm{~N} 80 \mathrm{M}$ \\
\hline Copper loading (\% by mass) & 76.2 & 74.6 & 79.2 \\
\hline Particulate & Nano & Nano : Micro & Nano : Micro \\
\hline Micro : nano particle \% & Nano only & $50: 50$ & $20: 80$ \\
\hline Primary solvent BP $\left({ }^{\circ} \mathrm{C}\right)$ & 117 & 136 & 136 \\
\hline
\end{tabular}

All three materials are similar show similar rheological behaviour with near rest viscosities in excess of 100 Pas, followed by shear thinning behaviour towards a shear thinning plateau approaching 30 Pas, Figure 1(c). This is typical of screen printing inks [29], [30] and their similarity in the rheological profiles would suggest that any variations in deposit between characteristics are not attributed to changes in the deposition process.

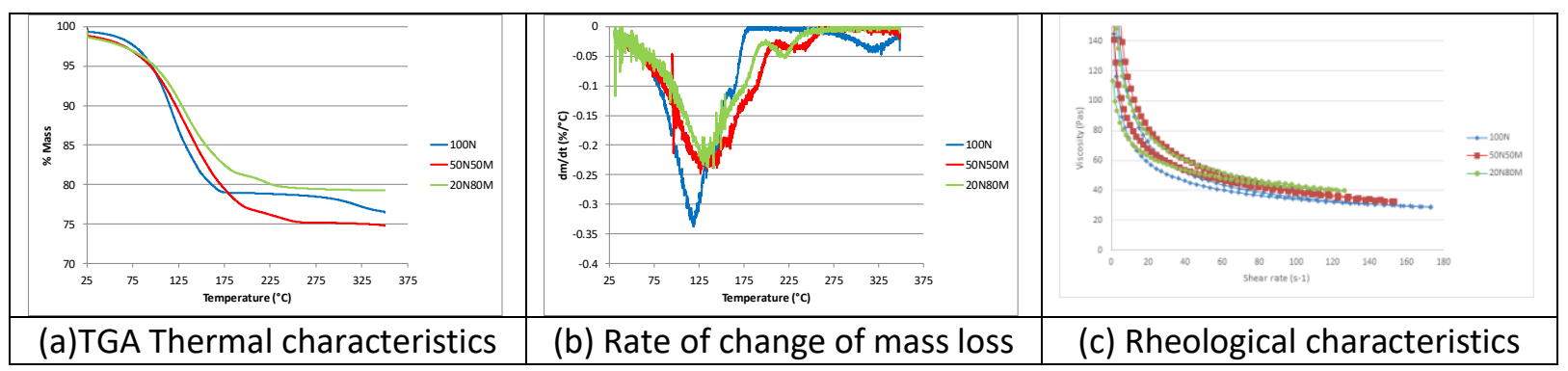




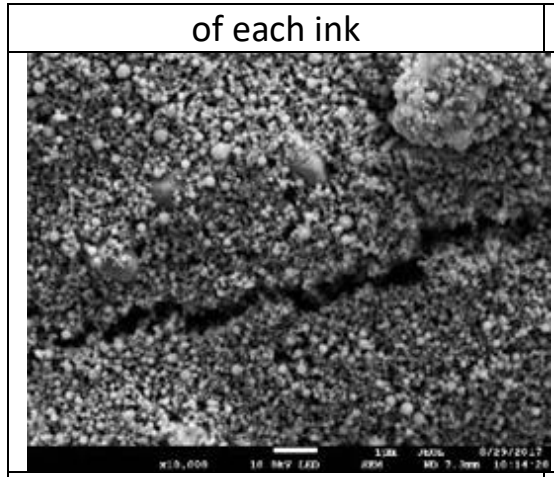

(d) $100 \mathrm{~N}$

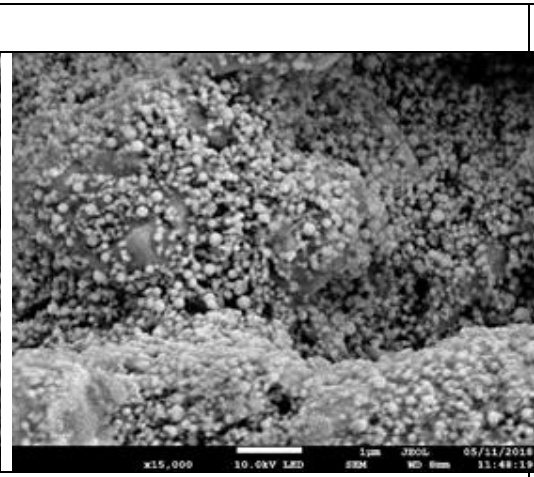

(e) $50 \mathrm{~N} 50 \mathrm{M}$

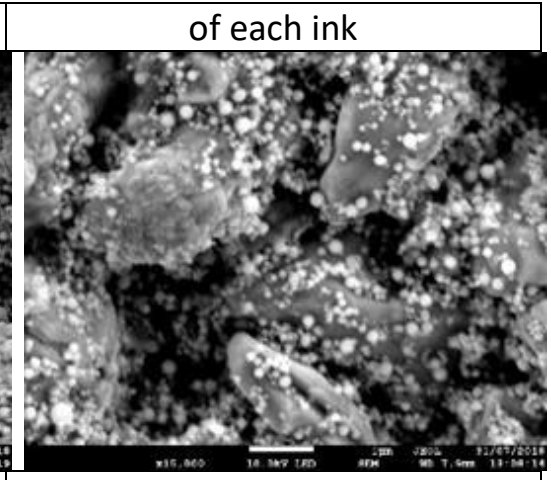

(f) $20 \mathrm{~N} 80 \mathrm{M}$

Figure 1 : (a \& b)Thermal Characteristics, (c) rheological and (d, e \& f) particle distribution of each of the copper inks. SEM images (d), (e) and (f) represent imaging of the dry but un-sintered films.

Each ink was screen printed using an image, Figure 2, which consisted of areas which allowed for characterization of fine lines ( $200 \mu \mathrm{m}-600 \mu \mathrm{m}$ wide), sheet resistance and contact resistance. This was printed to Tec-7 FTO coated glass from NSG (Nominal sheet resistance of $7 \Omega / \mathrm{sq}$ ), with a 110-34 mesh on an automatic ATMA flat bed printing machine. Prior to curing, each printed sample was dried in a Thieme hot dryer at $80^{\circ} \mathrm{C}$ for a residence time of 15 minutes. This allowed solvent evaporation to occur such that a dry film was obtained without the excessive temperature where nano-particle encapsulant would undergo sublimation. The printed substrates were cured in three atmospheres, inert $\mathrm{N}_{2}$ and reducing atmospheres using formic acid [31], [32] and a $5 \% \mathrm{H}_{2} / \mathrm{N}_{2}$, Figure 2(b). The time and time temperature of the formic acid were varied between 30 minutes and 120 minutes and between $175^{\circ} \mathrm{C}$ and $300{ }^{\circ} \mathrm{C}$. Under non reducing conditions, the FTO substrate has been shown to be stable at temperature $500^{\circ} \mathrm{C}$ [33] and thus would not be expected to change within the temperature range considered here.

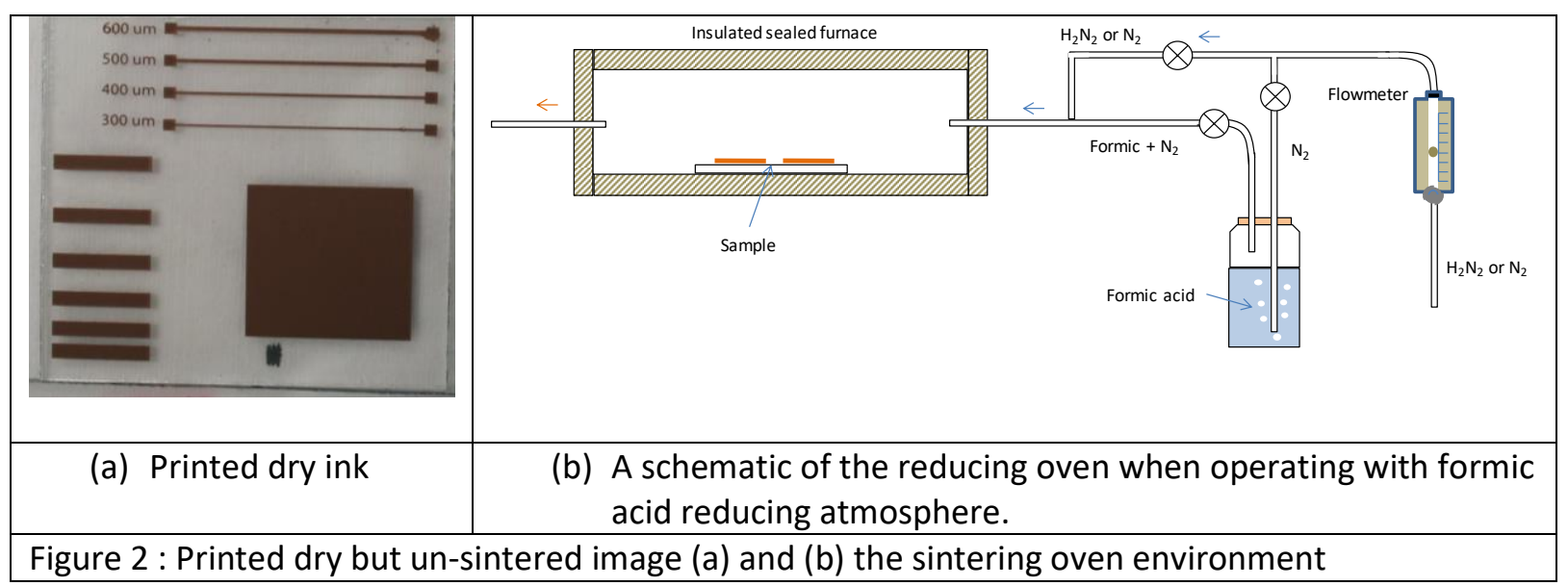

The resistance of the pattern structures was characterised with a Megger MIT 330-EN digital low resistance multimeter. As the copper is printed to the FTO, the measurement of line resistance therefore considers the parallel resistance of the printed ink and the underlying conductive FTO. Contact resistance and sheet resistance were calculated using the TLM method [34]. A Rofin 1064 $\mathrm{nm}$ Nd-YAG laser scribe was used for removal of the FTO was used to isolate the TLM area of the print. Three samples were produced at each condition and a mean taken. Within the three samples, line resistances were $+/-0.02 \Omega$, sheet resistance $0.04 \Omega$ /sq and contact resistance were $+/-0.44 \Omega$. 
The film thickness was measured using a Talysurf surftronic 100 stylus profileometer without signal filtering.

\section{Results.}

The thermal response of each material is significantly different and is also a function of the sintering environment. In comparing the line resistance of a nominally $600 \mu \mathrm{m}$ width line, the $100 \mathrm{~N}$ nano particle ink continuously produces the lowest line resistance, Figure $3(a-c)$, and is relatively insensitive to the sintering temperature in the $175^{\circ} \mathrm{C}-225^{\circ} \mathrm{C}$ range and extended exposure time. Above $225{ }^{\circ} \mathrm{C}$, extending the sintering time has a detrimental effect of the conductivity of the material with incremental increases in resistance with longer sintering time. Both the inks containing micro particles exhibit higher resistances than the nano particle ink, Figure 3 (d-f) \& Figure $3(\mathrm{~g}-\mathrm{i})$. In contrast to the $100 \mathrm{~N}$ ink, increasing sintering temperature reduces the resistance in each case. There is a more complex interaction between the sintering time and the resistance for micro - nano blend inks.

It is possible to sinter all three inks in a nitrogen environment, but the resistance of the printed line is reduced for those produced in a reducing environment is more consistent and controlled. Above $250{ }^{\circ} \mathrm{C}$ and 90 minutes, there was significant decolouration of the substrate as the high temperature acidic vapour (which had not thermally decomposed to $\mathrm{CO}$ and $\mathrm{H}_{2} \mathrm{O}$ ) reacted with the FTO coating (causing yellowing of the substrate), which resulted in delamination of the printed film. With the reducing atmosphere produced by the $5 \% \mathrm{H} 2 / \mathrm{N} 2$, the absolute values of the conductivity are comparable with that produced by the formic acid reducing atmosphere, although the high temperature sintering does not have such a detrimental effect on the FTO substrate.

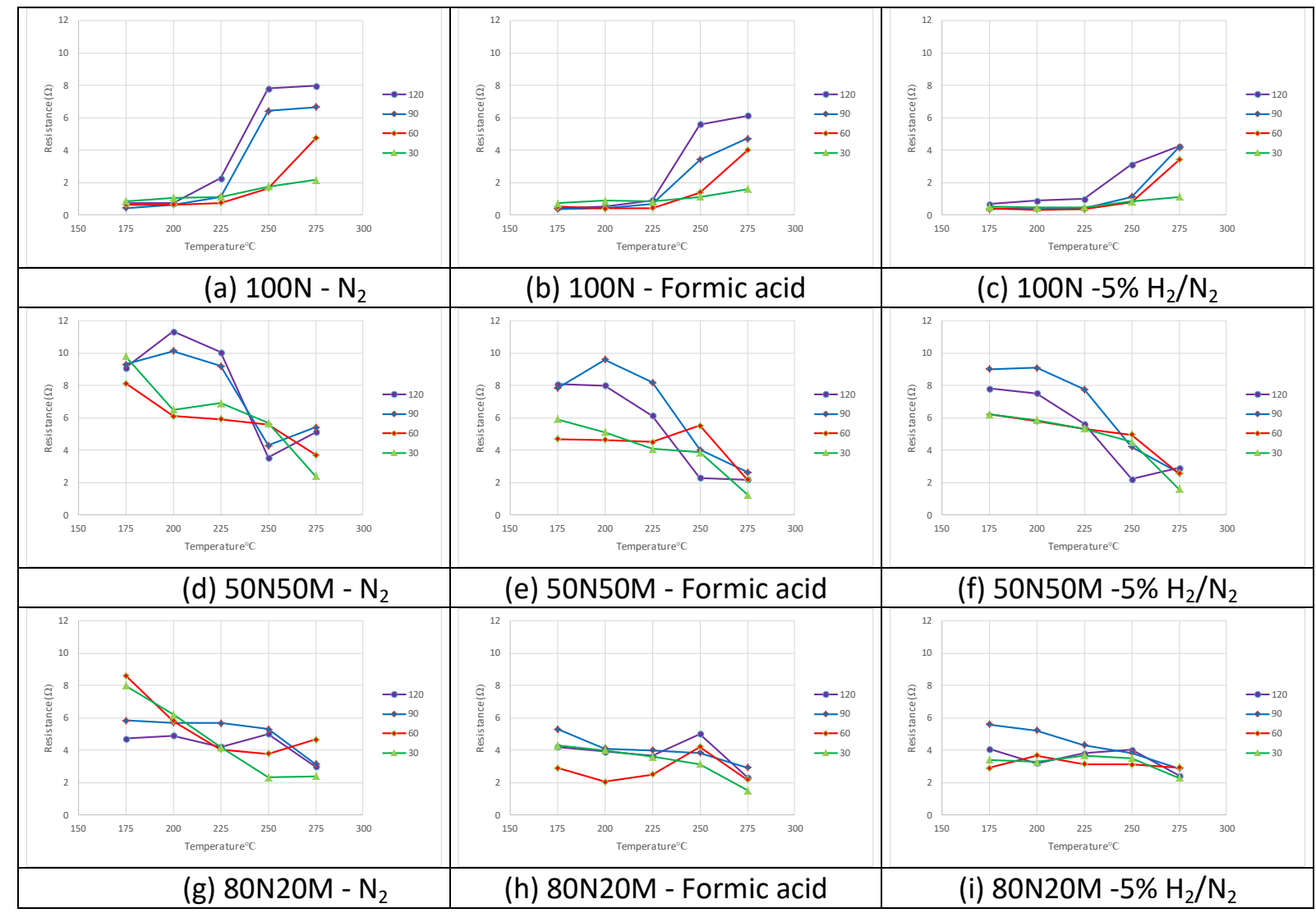


Figure 3 : Line resistance for a nominal $600 \mu \mathrm{m}$ wide line of $30 \mathrm{~mm}$ length under each sintering environment at residence times of $30,60,90$ and 120 minutes.

Similar behaviour to that observed with the line resistance is observed with the film sheet resistance, Figure $4(\mathrm{a}-\mathrm{i})$, although some differences are observed which have been attributed to geometrical nature of the feature being printed. For the $100 \mathrm{~N}$ ink, below $225^{\circ} \mathrm{C}$ the sheet resistance is mostly independent of the sintering time but extended times at higher temperatures result in significant increases in resistance, Figure $4(a-c)$. As with the line resistance, the negative impact of the higher temperatures is independent of the sintering atmosphere. Similar behaviour to resistance of the printed line was observed at below $225{ }^{\circ} \mathrm{C}$ for the sheet resistance of the $50 \mathrm{~N} 50 \mathrm{M}$ and $20 \mathrm{~N} 80 \mathrm{M}$ nano / micro inks. Above $250^{\circ} \mathrm{C}$, there is an increase in the sheet resistance at all sintering times for the 50N50M ink and the sensitivity to temperature and residence time reduces progressively with the quality of the reducing atmosphere. For the $20 \mathrm{~N} 80 \mathrm{M}$ ink, there is a more complex interaction between the sintering time and temperature, Figure 4 (g-i). At low sintering times, it is advantageous to sinter at a higher temperature, while an extended sintering time is detrimental at higher temperature.

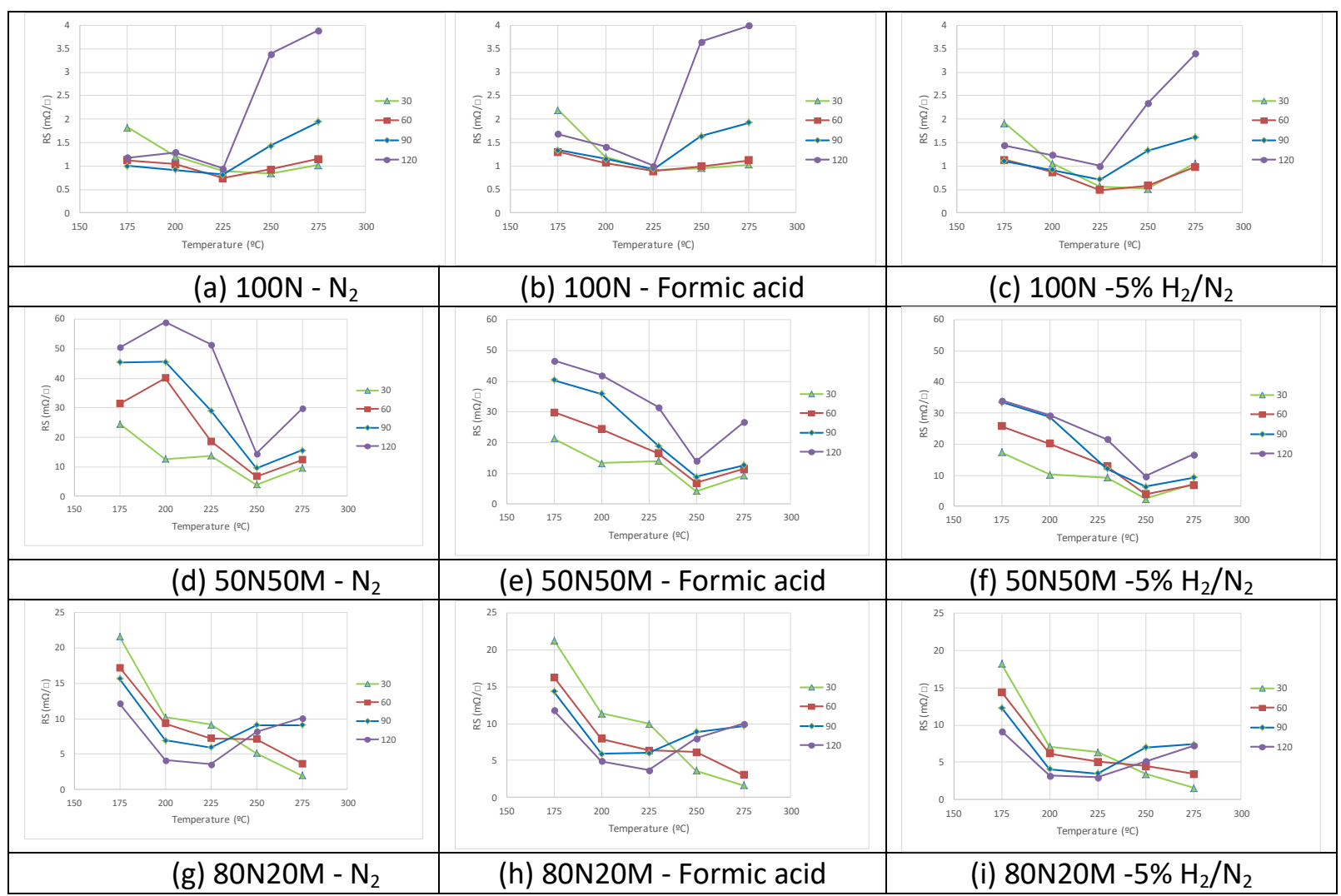

Figure 4 : Sheet resistance of each ink under the $\mathrm{N}_{2}$, Formic acid and $5 \% \mathrm{H}_{2} / \mathrm{N}_{2}$ sintering environements at residence times of $30,60,90$ and 120 minutes.

The trends observed in sheet resistance are generally repeated with the contact resistance, Figure 5 with typical contact resistances of between $0.5 \Omega$ and $12 \Omega$. The 120 minute sintering time has been 
omitted from the Figure as FTO / printed film damage was often observed leading to delamination causing significant variation in the data. Similarly the $100 \mathrm{~N}$ at $275{ }^{\circ} \mathrm{C}$ have been omitted as failure film adhesion failure occurred. For the $100 \mathrm{~N}$ material, minimum contact resistances are largely unaffected by sintering temperatures above $200{ }^{\circ} \mathrm{C}$ but can suffer a detrimental effect if held at the highest temperature for a prolonged period, Figure 5 (a-c). Contact resistances for the micro / nano particle inks are around 2 to 10 times that observed with the purely nano inks, Figure 5 (d-i). This is likely to be due to the reduced contact area interface associated with larger particles. For the $50 N 50 \mathrm{M}$ ink there is a general reduction in the contact resistance as the temperature is increased under all sintering conditions with a lower sintering time also consistently producing the minimum contact resistance. For the $20 \mathrm{~N} 80 \mathrm{M}$ ink, the contact resistance, is strongly affected by the sintering time in a nitrogen environment, but less so under reducing conditions. Under $5 \% \mathrm{H}_{2} / \mathrm{N}_{2}$ reducing conditions both the micro/nano blended materials exhibit the highest contact resistance.

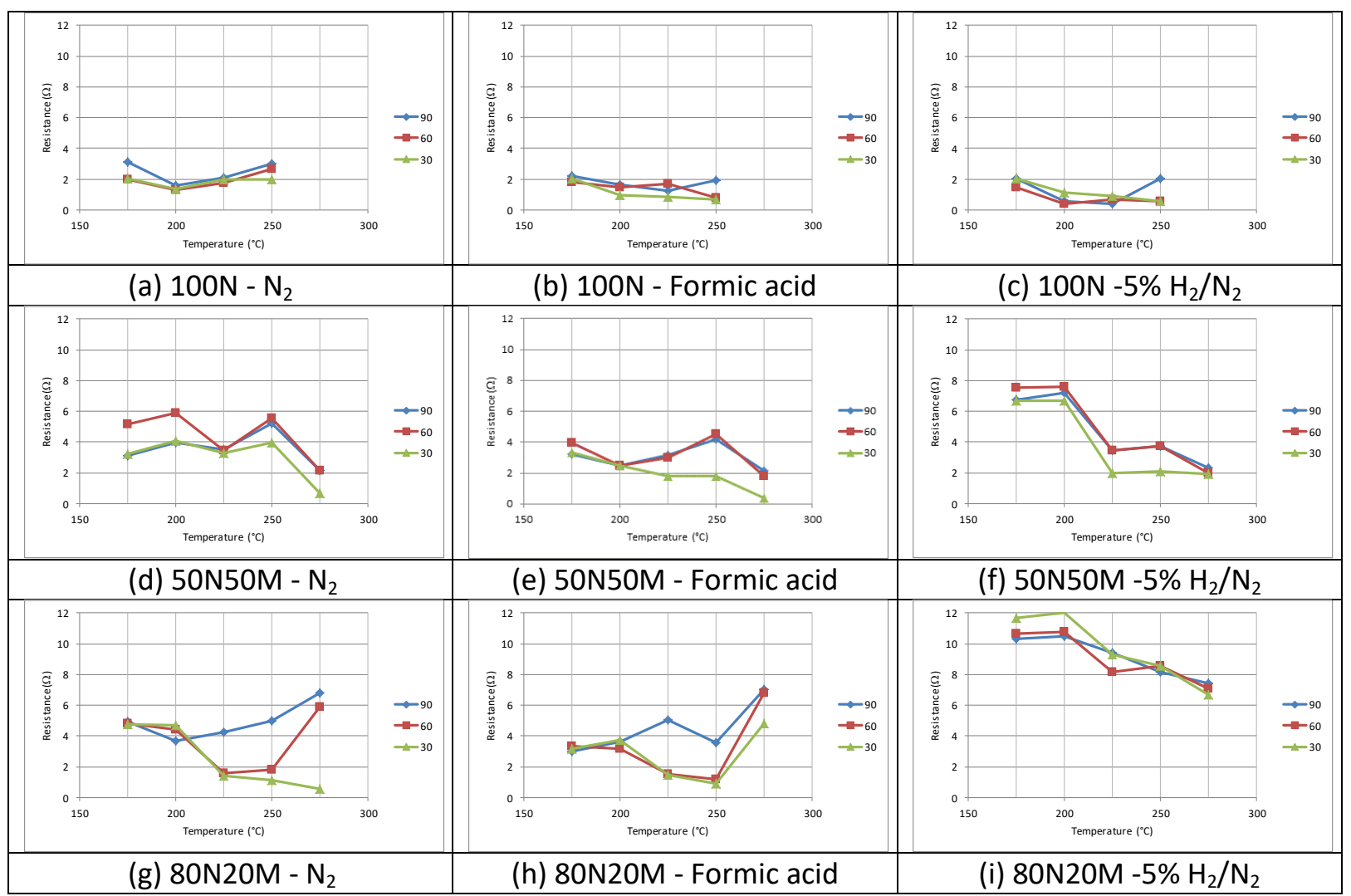

Figure 5 : Contact resistance of each ink under the N2, Formic acid and $5 \% \mathrm{H}_{2} / \mathrm{N}_{2}$ sintering environements at residence times of 30,60 and 90 .

The optimum sintering conditions (for minimum sheet resistance) for each material is summarized in Table 2. The overall benefit of sintering in a reducing atmosphere produced by formic acid compared to a nitrogen environment is minimal. Thus, given the relative ease by which a nitrogen environment can be achieved in a normal production environment, the more complex formic acid reducing environment is not recommended. There is a clear and consistent benefit of using the more controlled reducing atmosphere produced by the $5 \% \mathrm{H}_{2} / \mathrm{N}_{2}$ mixture. As the proportion of micro particles increases, there is an increase in the sintering temperature required. Overall the measured sheet resistance is between 2.2 to 8 times higher for the micro-nano blends inks than that obtained pure nano material ink. In comparison to the base resistivity of copper, the lowest obtainable 
resistivity is around 6 times that of copper for the $100 \mathrm{~N}$ ink. The micro - nano blended materials have a higher material resistivity, between 12 and 28 times that of base copper with lower resistivity under reducing conditions.

Table 2 : Optimum conditions and resultant sheet resistance and relative resistivity for each ink and atmosphere (Relative resistivity to copper at $\rho_{0=} 1.68 \times 10^{-8} \Omega \mathrm{m}$ )

\begin{tabular}{|l|c|c|c|c|c|c|c|c|c|c|c|c|}
\hline & \multicolumn{4}{|c|}{$\mathrm{N}_{2}$} & \multicolumn{4}{c|}{ Formic acid } & \multicolumn{5}{c|}{$5 \% \mathrm{H}_{2} / \mathrm{N}_{2}$} \\
\hline & $\mathrm{R}_{\mathrm{s}}$ & $\rho / \rho_{0}$ & $\mathrm{t}_{\text {opt }}$ & $\mathrm{T}_{\mathrm{opt}}$ & $\mathrm{R}_{\mathrm{s}}$ & $\rho / \rho_{0}$ & $\mathrm{t}_{\mathrm{opt}}$ & $\mathrm{T}_{\text {opt }}$ & $\mathrm{R}_{\mathrm{s}}$ & $\rho / \rho_{0}$ & $\mathrm{t}_{\text {opt }}$ & $\mathrm{T}_{\text {opt }}$ \\
\hline & $\mathrm{m} \Omega / \square$ & & $(\min )$ & $\left({ }^{\circ} \mathrm{C}\right)$ & $\mathrm{m} \Omega / \square$ & & $(\mathrm{min})$ & $\left({ }^{\circ} \mathrm{C}\right)$ & $\mathrm{m} \Omega / \square$ & & $(\mathrm{min})$ & $\left({ }^{\circ} \mathrm{C}\right)$ \\
\hline 100N & 0.74 & 7.3 & 60 & 225 & 0.89 & 6.3 & 60 & 225 & 0.49 & 5.8 & 60 & 225 \\
\hline 50N50M & 3.99 & 28 & 30 & 250 & 4.16 & 15 & 30 & 250 & 2.34 & 12 & 30 & 250 \\
\hline 20N80M & 1.99 & 29 & 30 & 275 & 1.65 & 19 & 30 & 275 & 1.61 & 16 & 30 & 275 \\
\hline
\end{tabular}

The sheet, line and contact resistances illustrated in Figures 3, 4 and 5 can be more readily examined from a mechanistic perspective by examination of SEM and XRD characteristics of the sintered films. Under ideal sintering conditions (minimum sheet resistance), the $100 \mathrm{~N}$ nano-particles sintered to form an interconnected network structure, Figure 6(a). The individual nano-particles exhibit melting and merge at the interface between the particles, indicating good degree of sintering Similar structures are obtained from sintered nano silver inks, [19], [35]. This particle network allows efficient charge transfer through the film. There is some intrinsic porosity in the film which has formed as a result of necking of the particles and the removal of the binder between the particles.

The $50 \mathrm{M} 50 \mathrm{~N}$ ink exhibits a structure where individual nano particles are bound intimately to the surface and between the micro particles, Figure $6(b)$, but the nano particles between the micro particles primarily remain as discrete spherical particles with minimal inter particle sintering. At the lowest nano particle concentration, there nano particles appear as discrete "nodules" which are bound to the micro particles which are in intimate contact with each other, Figure 6(c).

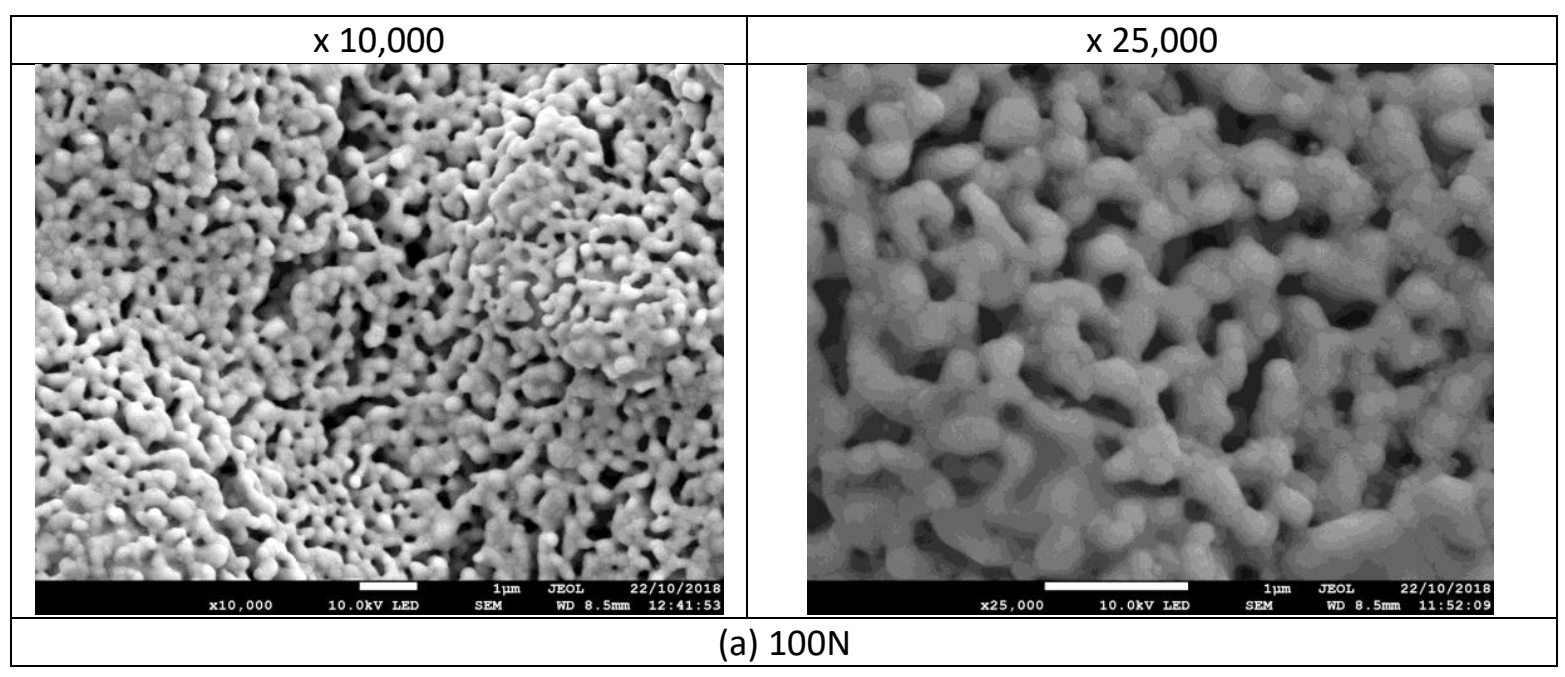




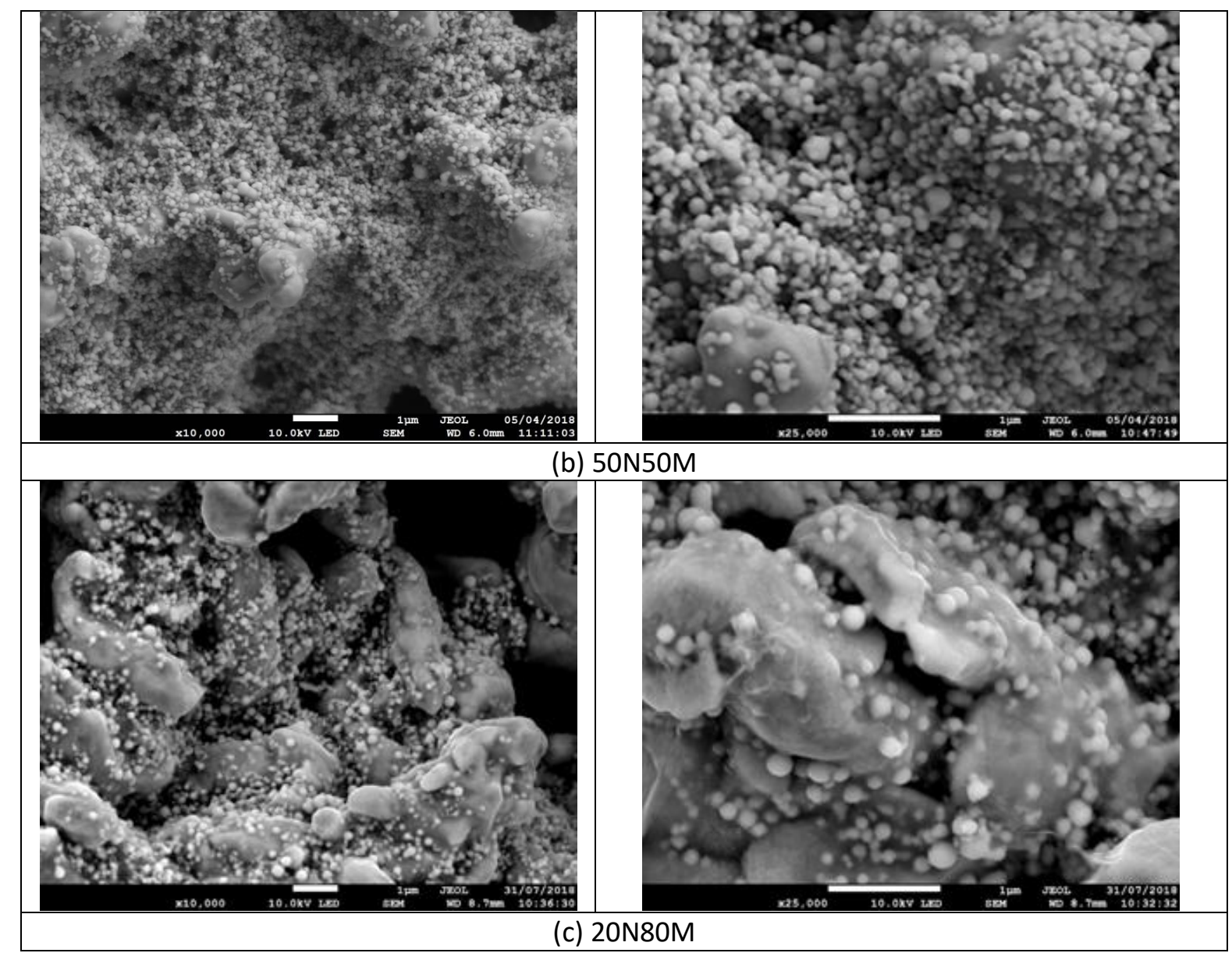

Figure 6 : SEM images of the sintered films for each ink at $\times 10,000$ and $x 25,000$ under ideal (minimum resistance condition).

XRD examination of dry printed copper films, Figures 7(a), (b) and (c) shows the three characteristic peaks at $43.2^{\circ}, 50.3^{\circ}$, and $74.1^{\circ}$ indicative of (111), (200), and (220), respectively. These characteristic peaks confirm that a face-centered cubic (FCC) copper phase is present. There is also evidence of the $\mathrm{Cu}_{2} \mathrm{O}$ phase at $36.6^{\circ}$ which indicates that some oxidation has occurred during the drying process [11], [36] for all three inks. When sintered for an hour in an $\mathrm{N}_{2}$ environment, all materials are dominated by the presence of the $\mathrm{Cu}$ peaks, although in case the $\mathrm{Cu}_{2} \mathrm{O}$ peak remains. The XRD spectra for the formic acid environment provide similar spectra to the nitrogen sintering environment which suggests that the $\mathrm{Cu}_{2} \mathrm{O}$ which is present in the dried sample does not undergo significant reduction during the formic acid sintering process. When sintered under a $5 \% \mathrm{H}_{2} / \mathrm{N}_{2}$ environment the $100 \mathrm{~N}$ and $50 \mathrm{~N} 50 \mathrm{M}$ material continue to contain $\mathrm{Cu}_{2} \mathrm{O}$, but this is absent for micro particle dominated ink, 20N80M. This suggests that the reducing conditions are conducive to the reduction of the $\mathrm{Cu}_{2} \mathrm{O}$ to $\mathrm{Cu}$, particularly as the temperature increases[37]. 


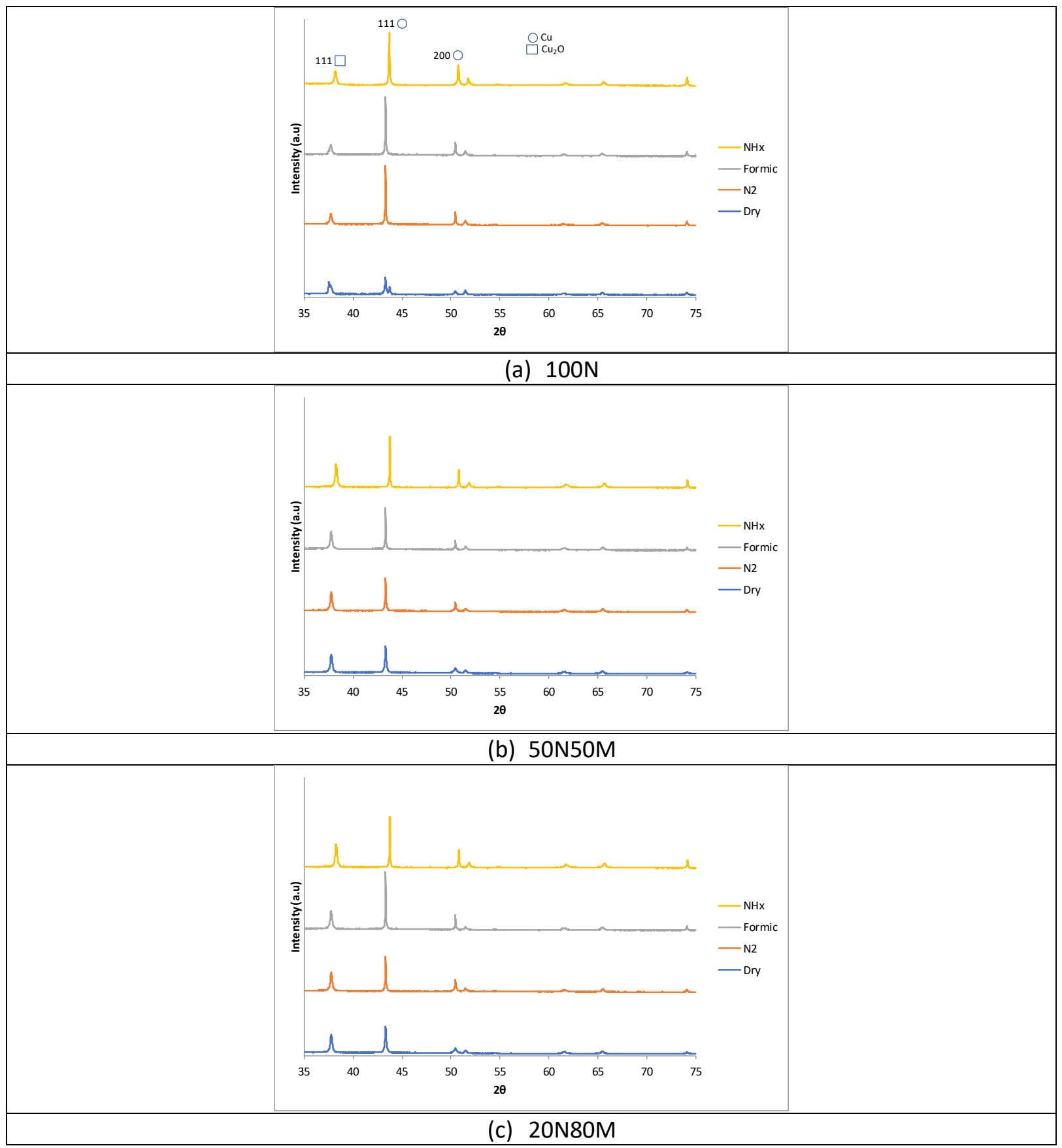

Figure 7 : XRD comparison of each film for each ink in a dry, N2 sintered for 60 min @ $200^{\circ} \mathrm{C}$, Formic acid for 60 min @ $200{ }^{\circ} \mathrm{C}$ and $5 \% \mathrm{H}_{2} / \mathrm{N}_{2}$ for 60 min @ $200{ }^{\circ} \mathrm{C}$.

Since oxidation is a source of changes in printed film resistance, samples were selected and measured immediately after sintering and then those sample samples were measured on a weekly basis when stored under a nominal environment of $21 \mathrm{C}+/-1^{\circ} \mathrm{C}, 60 \% \mathrm{RH}+/ 10 \%$, Figure 8 . In each instance the sample sintered for 30 minutes was chosen as all samples were blemish free at this sintering time. For the $100 \mathrm{~N}$ ink, there is a slight increase in resistance over time and in each instance, this change is largest at lower sintering temperatures, Figures 8(a)-(c). Of the three materials, the $50 \mathrm{~N} 50 \mathrm{M}$ material exhibits the most significant aging effect, Figures $8(\mathrm{~d})$ - $(\mathrm{g})$. There is an initial rise in resistance over the first week, followed by smaller increments over subsequent weeks and this behaviour is consistent for all sintering temperatures. For the 20N80M material 
exhibit minimal resistance increase over the period of measurement, inferring a very stable printed film, Figure Figures $8(\mathrm{~h})$-(j).

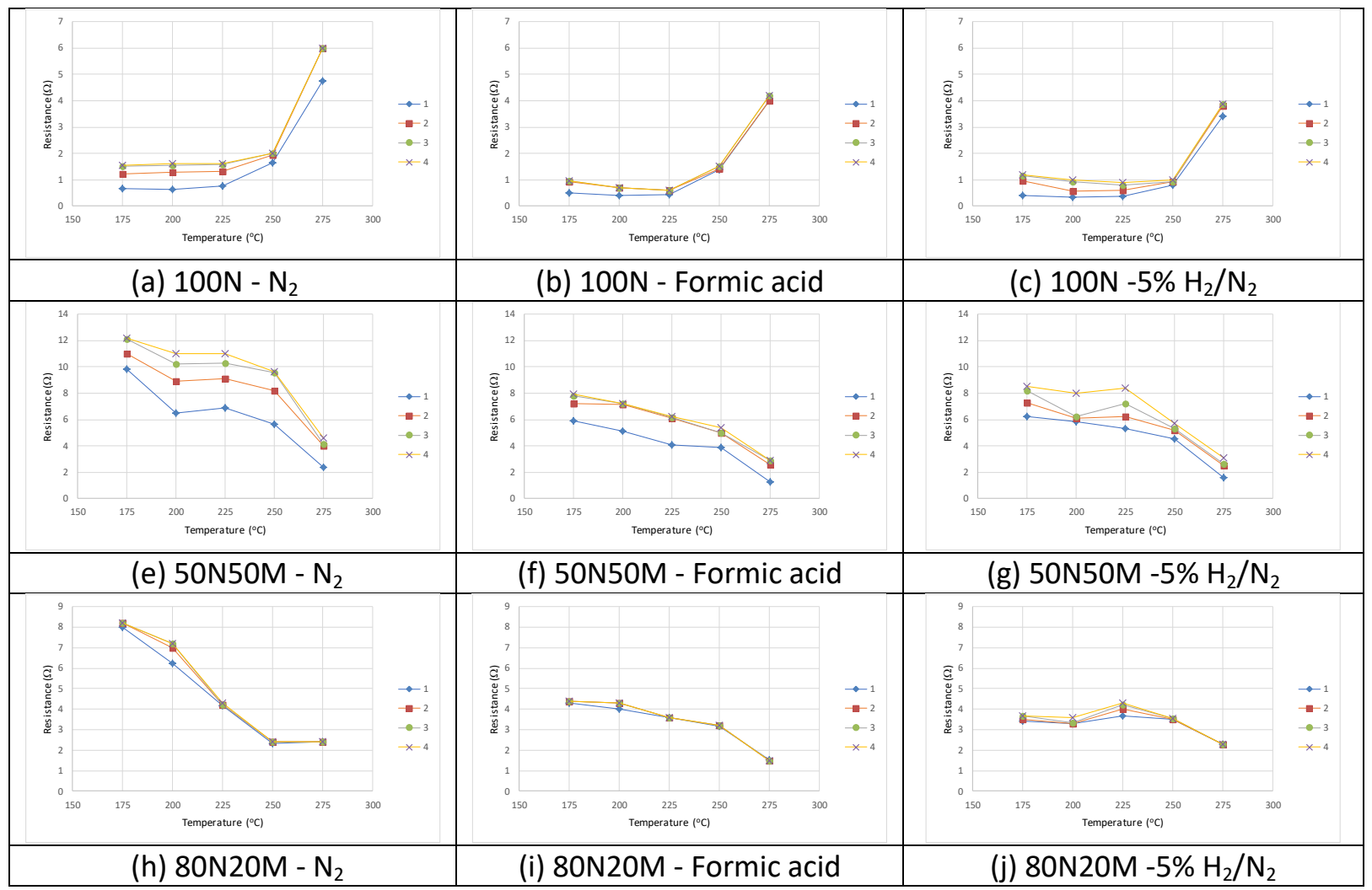

Figure 8 : Line resistance change over 4 week interval (labelled 1-4) for the three materials sintered at 30 minutes in each sintering environment.

\section{Discussion}

The relatively poor performance of the 50:50 nano particle : micro particle blend (highest sheet resistance, largest aging effect) suggests that there is an optimum blending ratio of nano / micro particles which balances the performance benefit of each constituent. It is postulated that the presence of an excessive quantity of micro particles disrupts the natural sintering tendency of the nano particles as they are rapidly bound to larger surface area micro particles, leaving them open to oxidation. Where the nano particles form a major contributor to the continuous conductive (50N50M) pathways, this impairs charge transfer through the particles. At lower concentrations of nano particles, a conductive pathway is ensured through micro particle contact and thus the impact of any nano particle oxidation is minimized. Evidence for this hypothesis can be seen in the absolute conductivity and also in the increase in resistance seen with aging, suggesting that there are some nano particles which are not fully sintered which are subject to oxidation. While the short term stability of the copper inks has been shown to be good when sintered under the correct conditions, further work should examine longer exposure periods under harsher thermal and moisture conditions which the conductive materials may be subjected during the lifetime of a product.

If printed copper lines were to be used to enhance the conductivity of a transparent surface (e.g. for charge extraction in PV), then it should be noted that the copper / FTO contact resistance is the same order of magnitude as the resistance of line $30 \mathrm{~mm}$ long $600 \mu \mathrm{m}$ wide line (Figure $3 \& 5$ ). The interfacial resistance component would form a significant portion of the the overall resistance. This 
is in contrast to that observed when copper plating from via magnetron spluttering [38] which would with FTO and copper having work functions of $4.4 \mathrm{eV}[39]$ and $4.7 \mathrm{eV}$ suggesting relatively unhindered charge transfer. This tends to indicate that the true contact area and contact chemistry is significantly more complex when using a printed ink. It also implies that any models for enhancing charge transfer through the use of printed mesh much take into account the contact resistance.

\section{Conclusions}

There is a significant interaction between the particles size distribution, the thermal sintering regime used and the subsequent characteristics of the final film when conductive copper inks are sintered on FTO coated glass substrates. The lowest resistances can be obtained using the pure nano copper ink and they also offer the advantage of a lower sintering temperature but a longer sintering time to achieve the lowest sheet resistance. Blending nano particles with micro particles can be disadvantageous in that it prevents full consolidate sintering of the nano particles, allowing them to be partially oxidized during the curing stage.

\section{Acknowledgements}

This work was made possible by the support given to the SPECIFIC Innovation and Knowledge Centre by the Engineering and Physical Science Research Council [EP/N020863/1], Innovate UK [920036], Engineering and Physical Science Research Council [EP/N509905/1] and by the European Regional Development Fund [c80892] through the Welsh Government. The authors would like to thank Hiprospects partner companies and institutions, in particular Intrinsiq materials for support in supplying materials

\section{References}

[1] Y. Kim, B. Lee, S. Yang, I. Byun, I. Jeong, and S. M. Cho, "Use of copper ink for fabricating conductive electrodes and RFID antenna tags by screen printing," Curr. Appl. Phys., vol. 12, no. 2, pp. 473-478, 2012.

[2] D. A. van den Ende, R. Hendriks, R. Cauchois, and W. A. Groen, "Large area photonic flash soldering of thin chips on flex foils for flexible electronic systems: In situ temperature measurements and thermal modelling," Electron. Mater. Lett., vol. 10, no. 6, pp. 1175-1183, 2014.

[3] S. Norita, D. Kumaki, Y. Kobayashi, T. Sato, K. Fukuda, and S. Tokito, "Inkjet-printed copper electrodes using photonic sintering and their application to organic thin-film transistors," Org. Electron., vol. 25, pp. 131-134, 2015.

[4] Y. S. Rosen, A. Yakushenko, A. Offenhäusser, and S. Magdassi, "Self-Reducing Copper Precursor Inks and Photonic Additive Yield Conductive Patterns under Intense Pulsed Light," ACS Omega, vol. 2, no. 2, pp. 573-581, 2017.

[5] A. Yabuki and S. Tanaka, "Electrically conductive copper film prepared at low temperature by thermal decomposition of copper amine complexes with various amines," Mater. Res. Bull., 
vol. 47, no. 12, pp. 4107-4111, 2012.

[6] B. Lee, Y. Kim, S. Yang, I. Jeong, and J. Moon, "A low-cure-temperature copper nano ink for highly conductive printed electrodes," Curr. Appl. Phys., vol. 9, no. 2 SUPPL., pp. e157-e160, 2009.

[7] Y. Lee, J.-R. Choi, K. J. Lee, N. E. Stott, and D. Kim, "Large-scale synthesis of copper nanoparticles by chemically controlled reduction for applications of inkjet-printed electronics," Nanotechnology, vol. 19, no. 41, p. 415604, 2008.

[8] E. B. Jeon, S. J. Joo, H. Ahn, and H. S. Kim, "Two-step flash light sintering process for enhanced adhesion between copper complex ion/silane ink and a flexible substrate," Thin Solid Films, vol. 603, pp. 382-390, 2016.

[9] W. H. Chung, H. J. Hwang, and H. S. Kim, "Flash light sintered copper precursor/nanoparticle pattern with high electrical conductivity and low porosity for printed electronics," Thin Solid Films, vol. 580, pp. 61-70, 2015.

[10] S. Magdassi, M. Grouchko, and A. Kamyshny, "Copper nanoparticles for printed electronics: Routes towards achieving oxidation stability," Materials (Basel)., vol. 3, no. 9, pp. 4626-4638, 2010.

[11] S. Choudhary et al., "Oxidation mechanism of thin Cu films: A gateway towards the formation of single oxide phase," AIP Adv., vol. 8, no. 5, 2018.

[12] K. Ida et al., "Behavior of Cu nanoparticles ink under reductive calcination for fabrication of Cu conductive film," Thin Solid Films, vol. 520, no. 7, pp. 2789-2793, 2012.

[13] D. Deng, Y. Jin, Y. Cheng, T. Qi, and F. Xiao, "Copper nanoparticles: Aqueous phase synthesis and conductive films fabrication at low sintering temperature," ACS Appl. Mater. Interfaces, vol. 5, no. 9, pp. 3839-3846, 2013.

[14] A. Soltani, B. Khorramdel Vahed, A. Mardoukhi, and M. Mäntysalo, "Laser sintering of copper nanoparticles on top of silicon substrates," Nanotechnology, vol. 27, no. 3, p. 035203, 2016.

[15] D. Lopez-Espiricueta, E. Fearon, S. Edwardson, and G. Dearden, "Direct Write Processing of Multi-micron Thickness Copper Nano-particle Paste on Flexible Substrates with $532 \mathrm{~nm}$ Laser Wavelength," Phys. Procedia, vol. 83, pp. 194-203, 2016.

[16] M. Joo, B. Lee, S. Jeong, and M. Lee, "Laser sintering of Cu paste film printed on polyimide substrate," Appl. Surf. Sci., vol. 258, no. 1, pp. 521-524, 2011.

[17] M. Aarnts et al., "Effect of substrate roughness on tin layer performance after reflow Surface investigation on the so called Roughness Matrix - TSP IJW trial P0514 Reference Source number : Project number : Date of issue : Security Code : 30 October 2015 Author ( s ): Joos," 2015.

[18] K. Eun, M. W. Chon, T. H. Yoo, Y. W. Song, and S. H. Choa, "Electromechanical properties of printed copper ink film using a white flash light annealing process for flexible electronics," Microelectron. Reliab., vol. 55, no. 5, pp. 838-845, 2015.

[19] W. H. Chung, H. J. Hwang, S. H. Lee, and H. S. Kim, "In situ monitoring of a flash light sintering process using silver nano-ink for producing flexible electronics," Nanotechnology, vol. 24, no. 3, 2013. 
[20] H. J. Hwang, K. H. Oh, and H. S. Kim, "All-photonic drying and sintering process via flash white light combined with deep-UV and near-infrared irradiation for highly conductive copper nano-ink," Sci. Rep., vol. 6, no. May 2015, pp. 1-10, 2016.

[21] C. H. Ryu, S. J. Joo, and H. S. Kim, "Two-step flash light sintering of copper nanoparticle ink to remove substrate warping," Appl. Surf. Sci., vol. 384, pp. 182-191, 2016.

[22] M. M. Haque, D. Cho, and C. S. Lee, "Investigation of sintering behavior of octanethiol-coated copper nano ink under various atmospheres," Thin Solid Films, vol. 536, pp. 32-38, 2013.

[23] M. Joo, B. Lee, S. Jeong, and M. Lee, "Comparative studies on thermal and laser sintering for highly conductive Cu films printable on plastic substrate," Thin Solid Films, vol. 520, no. 7, pp. 2878-2883, 2012.

[24] F. De Rossi et al., "All Printable Perovskite Solar Modules with $198 \mathrm{~cm}^{2}$ Active Area and Over 6\% Efficiency," Adv. Mater. Technol., vol. 3, no. 11, p. 1800156, Nov. 2018.

[25] J. Baker et al., "High throughput fabrication of mesoporous carbon perovskite solar cells," J. Mater. Chem. A, vol. 5, no. 35, pp. 18643-18650, 2017.

[26] Y. Galagan et al., "Evaluation of ink-jet printed current collecting grids and busbars for ITOfree organic solar cells," Sol. Energy Mater. Sol. Cells, vol. 104, pp. 32-38, 2012.

[27] Y. Cai, L. Liang, and P. Gao, "Promise of commercialization: Carbon materials for low-cost perovskite solar cells," Chinese Phys. B, vol. 27, no. 1, 2018.

[28] N. L. Chang, A. W. Yi Ho-Baillie, P. A. Basore, T. L. Young, R. Evans, and R. J. Egan, "A manufacturing cost estimation method with uncertainty analysis and its application to perovskite on glass photovoltaic modules," Prog. Photovoltaics Res. Appl., vol. 25, no. 5, pp. 390-405, 2017.

[29] E. H. Jewell, S. M. Hamblyn, T. C. Claypole, and D. T. Gethin, "The impact of carbon content and mesh on the characteristics of screen printed conductive structures," Circuit World, vol. 39, no. 1, pp. 13-21, Feb. 2013.

[30] H. Lin, C. Chang, W. Hwu, and M. Ger, "The rheological behaviors of screen-printing pastes," J. Mater. Process. Technol., vol. 197, no. 1-3, pp. 284-291, Feb. 2008.

[31] B. Y. Wang, T. H. Yoo, Y. W. Song, D. S. Lim, and Y. J. Oh, "Cu ion ink for a flexible substrate and highly conductive patterning by intensive pulsed light sintering," ACS Appl. Mater. Interfaces, vol. 5, no. 10, pp. 4113-4119, 2013.

[32] Y. Gao et al., "Novel copper particle paste with self-reduction and self-protection characteristics for die attachment of power semiconductor under a nitrogen atmosphere," Mater. Des., vol. 160, pp. 1265-1272, 2018.

[33] K. Hooper, M. Carnie, C. Charbonneau, and T. Watson, "Near infrared radiation as a rapid heating technique for $\mathrm{TiO} 2$ films on glass mounted dye-sensitized solar cells," Int. J. Photoenergy, vol. 2014, no. 1, 2014.

[34] W. G. O. J.G.J Gern, "IEEE Electron Device Letters," IEEE Electron Device Lett., vol. 40, no. 1, pp. C2-C2, Jan. 2019.

[35] C. L. Lee, K. C. Chang, and C. M. Syu, "Silver nanoplates as inkjet ink particles for metallization at a low baking temperature of $100^{\circ} \mathrm{C}$," Colloids Surfaces A Physicochem. Eng. Asp., vol. 381, 
no. 1-3, pp. 85-91, 2011.

[36] S. Choudhary, J. V. N. Sarma, and S. Gangopadhyay, "Growth and characterization of single phase Cu2O by thermal oxidation of thin copper films," AIP Conf. Proc., vol. 1724, no. April 2016, 2016.

[37] J. Y. Kim, J. A. Rodriguez, J. C. Hanson, A. I. Frenkel, and P. L. Lee, "Reduction of CuO and Cu2O with $\mathrm{H} 2$ : $\mathrm{H}$ embedding and kinetic effects in the formation of suboxides," J. Am. Chem. Soc., vol. 125, no. 35, pp. 10684-10692, 2003.

[38] U. D. Ab, R. Z. J. Ziegler, L. Xia, and D. Borchert1 1, "Changes in Contact Resistance of Different Metals To Magnetron Sputtered Ito," 25th Eur. PV Sol. Energy Conf., no. September, pp. 1740-1743, 2010.

[39] M. G. Helander, M. T. Greiner, Z. B. Wang, W. M. Tang, and Z. H. Lu, “Work function of fluorine doped tin oxide," J. Vac. Sci. Technol. A Vacuum, Surfaces, Film., vol. 29, no. 1, p. 011019, 2011. 